\title{
Germanica
}

\section{Personnages juifs dans le Zeitroman de la République de Weimar}

Jüdische Gestalten im Zeitroman

\section{Anne Lagny}

\section{(2) OpenEdition}

\section{Journals}

Édition électronique

URL : http://journals.openedition.org/germanica/2389

DOI : 10.4000/germanica.2389

ISSN : 2107-0784

Éditeur

Université de Lille

Édition imprimée

Date de publication : 31 décembre 1991

Pagination : 145-168

ISSN : 0984-2632

\section{Référence électronique}

Anne Lagny, "Personnages juifs dans le Zeitroman de la République de Weimar », Germanica [En ligne], 9 | 1991, mis en ligne le 15 juillet 2014, consulté le 06 octobre 2020. URL : http:// journals.openedition.org/germanica/2389; DOI : https://doi.org/10.4000/germanica.2389

Ce document a été généré automatiquement le 6 octobre 2020.

(C) Tous droits réservés 


\section{Personnages juifs dans le Zeitroman de la République de Weimar}

Jüdische Gestalten im Zeitroman

Anne Lagny

Pour Jean Weinberg

1 Une première constatation s'impose, à la lecture du Zeitroman weimarien : celle de la place réduite qu'y occupe le thème juif. La différence est grande avec des œuvres comparables de la période précédente, où le personnage juif, omniprésent, est construit comme pôle de répulsion de la germanité1. Comment rendre compte de l'éclipsé relative du personnage juif? La réponse à cette question suppose que nous ne cherchions pas dans des œuvres de fiction un simple reflet de la réalité contemporaine. Il est aisé, certes, de mettre en rapport la normalisation de l'image du juif avec les progrès de l'assimilation durant cette période (on sait que la levée des dernières interdictions légales marque une étape décisive de l'intégration des juifs dans la société allemande, même si la survivance d'une discrimination fait apparaître la condition juive comme le produit contradictoire de «l'assimilation et de la discrimination ») 2 . Mais nous borner à repérer de simples convergences avec les résultats de l'analyse économique et sociologique serait réduire la signification du personnage (juif) à une simple question de vérité documentaire, alors qu'elle fait intervenir le problème de la figuration littéraire.

Il ne s'agit donc pas, pour l'essentiel, de rassembler des informations sur l'antisémitisme, ou éventuellement sur les métiers exercés par les juifs, leur insertion dans la société et les obstacles qu'ils y rencontrent ${ }^{3}$, mais de rendre compte d'une certaine difficulté de figuration de la réalité juive sous la République de Weimar. En conséquence, nous avons préféré substituer à une énumération de motifs récurrents de la figuration du juif ou de l'antisémitisme une étude plus approfondie de types juifs dans leur contexte romanesque (Denn sie wissen, was sie tun, de Ernst Ottwalt; Erfolg, de Lion Feuchtwanger et Die Stadt, de Ernst von Salomon). L'analyse du statut du 
personnage juif dans le roman peut en effet faire progresser notre connaissance, dans la mesure où elle fait droit à la spécificité du matériau littéraire, d'une lisibilité moins immédiate que l'essai ou le témoignage autobiographique.

Quelques observations préalables, même brèves, sur la structure et le fonctionnement $\mathrm{du}$ genre feront mieux cerner ses possibilités et ses limites de figuration. Conformément à l'usage, nous entendons par «Zeitroman», ou roman d'époque, des œuvres qui décrivent une réalité contemporaine de celle que vivent leurs auteurs. C'est le genre dans lequel l'époque se fait roman, tente de parvenir à la conscience d'ellemême ${ }^{4}$. Intégrant l'actualité comme une donnée essentielle, il en figure les thèmes principaux ou secondaires sous l'aspect de crises et de conflits non résolus. Il est l'un des genres les plus aptes, peut-être, à réfracter la réalité d'une époque en travail, dans sa dimension multiple, confuse, en épousant la perspective de la conscience immergée dans le Jetztzeit. De ce point de vue, le Zeitroman, genre de l'époque ouverte, du sens qui n'est pas encore constitué, ne se réduit pas à une variante du roman historique ${ }^{5}$. Celuici en effet prend pour cadre une époque révolue, close sur elle-même, dont la lisibilité lui permet de figurer esthétiquement la résolution d'une problématique, même contemporaine $^{6}$.

Un exemple nous suffira pour suggérer l'impact de ces réflexions sur le sujet qui nous intéresse. Deux œuvres de Lion Feuchtwanger, le roman historique Jud Süß et le roman d'époque Erfolg, thématisent la réalité juive et l'antisémitisme sous la République de Weimar, mais la réfraction de cette même problématique contemporaine dans deux genres distincts se traduit par une différence dans la figuration du personnage. Le roman historique nous présente un héros glorieux, qui choisit librement son destin de juif (alors qu'il pourrait s'en affranchir, lorsqu'il apprend qu'il est le fils illégitime d'un Allemand) et dont le supplice ignominieux, apparemment incompatible avec l'intégrité de l'individu, scelle l'appartenance au peuple élu et marque le triomphe du judaïsme comme principe spirituel. L'avocat juif Siegbert Geyer de Erfolg, en revanche, est impuissant à transformer la fatalité de sa condition en destin assumé: victime d'agressions racistes ordinaires, qui minent son intégrité physique et mentale, exclu de la communauté allemande et du pouvoir réel, il est aliéné par le présent, contraint à des compromissions et des abdications qui épuisent le sens de son existence individuelle. Là où le roman historique conclut sur la vision idéale de la communauté juive escortant le cercueil de Süß, Erfolg s'achève sur l'exil solitaire de Geyer, dont le nom est effacé des œuvres qui fixent son époque en histoire.

Le Zeitroman, dans la mesure où il n'impose pas le point de vue d'une résolution artificielle, nous permettra d'évaluer la place et l'importance du thème juif dans la conscience d'époque. Le choix d'auteurs d'orientations politiques différentes (le communisme, avec Ottwalt, l'intelligence libérale, avec Feuchtwanger, et la révolution conservatrice, avec Salomon) montrera que le système d'interprétation du fait juif et de l'antisémitisme conditionne le degré plus ou moins élevé de figuration des différents motifs juifs ou antisémites, et donc le découpage du complexe de faits romanesque.

\section{Genia Lazar, juive, femme et communiste}

6 Le roman de Ottwalt, Denn sie wissen, was sie tun, est l'un des plus violents réquisitoires contre la Klassenjustiz de Weimar et la caste des juges, dont la partialité évidente dans les procès est conditionnée par le « ressentiment ». Menacés dans leur position sociale 
et économique après la guerre, hantés par le spectre du déclassement («Nous allons descendre au niveau de la canaille! Quelle misère!») (92) ils organisent leur "défense " contre la République (" construire des digues contre le flot boueux de l'incroyance et de l'injustice» (29), celle qui a ébranlé leur position) et le prolétariat, accusé d'avoir détruit l'ordre social et corrompu les valeurs morales?

7 Le roman décrit les stations de la carrière du juge Dickmann, en reconstituant l'enchaînement par lequel un homme médiocre, «produit de son éducation, de sa caste, du système » (ainsi que le définit Kurt Tucholsky dans sa recension du roman) est conditionné pour devenir un des rouages de la machine judiciaire qui écrase et qui tue. Impuissant à réfléchir ses déterminations économiques et sociales, otage du système, le personnage finit par s'adapter, au prix d'une insensibilisation progressive à toute conscience, morale et politique, et de quelques disfonctionnements, banalisés en troubles psychologiques ${ }^{8}$.

8 L'épisode de la liaison avec Genia Lazar, au centre du roman, coïncide avec la période de désorientation qui s'étend jusqu'à la fin des années de formation de Dickmann. Jeune juriste stagiaire, de bonne famille bourgeoise, militante communiste, Genia est intellectuellement et moralement supérieure à Dickmann, à qui elle donne une clé de compréhension de sa situation en termes de matérialisme historique. Elle exige de lui un engagement politique, sans croire qu'il en soit capable: «Tu n'as fait que perdre le courage d'être injuste, alors que tes amis, eux, ont ce courage » (213). Le départ de Dickmann, nommé juge assesseur dans un fief de la réaction, consacre le triomphe du milieu réactionnaire, mais la rupture n'a pas pour Genia de signification humiliante, et laisse ses convictions et le sens de son existence intacts.

9 Sans intérêt au plan psychologique ou dramatique, l'épisode figure la mise en accusation de la mentalité réactionnaire par la communiste, porte-parole de l'auteur, qui introduit donc le point de vue d'une intelligibilité supérieure. Au moment où Dickmann va basculer du côté de la réaction, elle explicite les conséquences de sa décision (rester dans le système, c'est se faire le complice de la répression et de l'injustice), dénonce ses faux-fuyants et démasque sa «personnalité autoritaire ».

La fonction essentielle du roman - l'éducation politique et l'appel à l'engagement - est donc confiée à un personnage de femme, juive et communiste, susceptible de faire apparaître par contraste le système de l'idéologie réactionnaire en suscitant une triple réaction de défense: antisémitisme, misogynie, anticommunisme. Elle est le porteparole de la critique la plus violente de l'institution judiciaire ${ }^{9}$, relayée à la fin du roman par un avocat, juif lui aussi, qui prononce la dernière condamnation de la justice de classe, dans un contexte désormais acquis à la réaction. Cette image positive du juif militant pour la justice sociale s'accorde avec celles que donnent d'autres Justizromane de la République de Weimar. Le type de l'avocat juif militant, souvent isolé dans un milieu réactionnaire, est un topos de cette littérature.

Le personnage juif n'apparait donc pas ici en tant que tel, mais comme représentant ou allié du communisme ${ }^{10}$. Genia Lazar ne se définit jamais en tant que femme, ou juive. De même, l'antisémitisme n'est pas dissocié ici de la mentalité réactionnaire, dont il est une composante au même titre que les sympathies monarchistes et la haine de la République, le ressentiment contre les ouvriers, l'anticommunisme, la misogynie ${ }^{11}$, la défense des "valeurs ». Expression de la réaction politique et sociale, figurée dans des types sociaux appartenant à des milieux tels que la haute magistrature, l'université, l'aristocratie des Junkers, il est entièrement réductible à l'anticommunisme, dont il est 
la manifestation inactuelle: l'ouvrier et le pauvre sont aujourd'hui pour le réactionnaire ce qu'était le juif, hier, pour l'antisémite ${ }^{12}$. Le modèle d'explication communiste, fonctionnant ici comme filtre, conditionne le découpage d'un complexe romanesque dans lequel ne trouvent pas place les thèmes proprement juifs, tels que l'identité juive, l'assimilation, et qui ne retient qu'un type d'antisémitisme, celui des milieux anticommunistes.

\section{Siegbert Geyer dans la « foire aux vanités » de la justice bavaroise}

12 Dans Erfolg, Feuchtwanger dépeint le triomphe de la réaction politique dans la Bavière des années 1921-1924, écartelée entre archaïsme et modernité. La multiplication des foyers de narration traduit le souci de composer un panorama d'époque, alors que Ottwalt, plus proche $\mathrm{du}$ reportage, limite son roman au découpage d'un Tatsachenkomplex: Erfolg est tout d'abord le Justizroman bavarois, pour lequel Feuchtwanger s'est inspiré librement de faits réels de la chronique judiciaire de l'époque ${ }^{13}$; par ailleurs, l'auteur a figuré sous des noms d'emprunt l'actualité politique de ces trois années, l'agitation de l'extrême-droite, favorisée par le gouvernement réactionnaire de Bavière, la constitution du parti nazi (les «Wahrhaft Deutsche» de Kutzner, alias Hitler) et le putsch manqué ${ }^{14}$; enfin la réflexion sur l'art et la place de l'intellectuel dans la société définit la perspective dominante à la fin du roman. La résolution des conflits de l'époque, toujours ouverts au plan politique, s'accomplit au niveau artistique, et donne à l'ensemble une conclusion apaisée.

13 Rattaché aux intrigues et aux dimensions essentielles du roman, l'avocat Siegbert Geyer, présent du début à la fin, est l'un des personnages les plus fortement caractérisés, mais, à la différence de tous les autres, c'est celui qui échoue le plus totalement. Trois contradictions fondamentales, insolubles pour lui, conditionnent sa déchéance finale :

14 - le droit contre la force : avocat de la défense, il s'oppose vainement à l'ordre établi et n'obtient pas la révision du procès. Par une ironie du sort, c'est un riche industriel qui marchande la relaxation du prisonnier ;

15 - la raison contre le sentiment: député social-démocrate, il ne peut faire une interpellation au Reichstag contre le scandale de la réaction en Bavière car son fils, impliqué dans un Fememord, et secrétaire des Wahrhaft Deutsche, l'a compromis avec lui, ruinant ainsi son crédit politique ;

16 - l'action contre la réflexion: son projet d'élaborer la théorie d'une réforme politique est abandonné au profit d'une action quotidienne obscure.

17 Ces contradictions se résument dans la dialectique entre les deux principes du pouvoir (Macht) et de l'esprit (Geist), qui s'incarne dans le conflit entre le ministre de la justice bavarois Otto Klenk et l'avocat Siegbert Geyer. Le contraste physique entre la vitalité d'Otto Klenk (Bavarois solide, bon vivant) et la fragilité de Geyer (l'intellectuel rongé par la nervosité) traduit l'inégalité du rapport de forces. L'opposition de l'avocat, cantonnée au discours et à la défense des principes, ne menace pas sérieusement le pouvoir en place. Mais Klenk, représentant d'une Realpolitik bornée et sans scrupules, a néanmoins sur Geyer une supériorité positive : sa connaissance des hommes, condition de son efficacité, alors que l'avocat, d'une lucidité supérieure, reste l'homme des 
abstractions. "Geyer comprenait quelque chose à la justice et à l'humanité, mais il ne comprenait rien à l'homme en particulier. Il n'était pas impossible que Klenk eût raison, ce qui remplissait l'avocat d'une rage impuissante » (153).

Le personnage de Siegbert Geyer incarne dans le roman la défaite de l'esprit. Alors qu'il jouait un rôle de premier plan au début du roman, il est, à la fin, réduit au silence et à l'insignifiance. Le pôle de l'esprit opposé à Klenk, retiré du pouvoir, est occupé désormais par le journaliste Tüverlin, qui, à l'inverse de Geyer, n'a pas émoussé les armes de l'esprit dans un combat vain contre la force, mais qui, sur la base de la foi en la raison, prédit la victoire morale de l'esprit dans l'histoire: "Les grands empires passent, un bon livre reste » (761). Avec Johanna Krain, la jeune femme enracinée dans le Land de Bavière, il attend que vienne le temps de l'histoire pour écrire des œuvres qui portent témoignage sur la réaction en Bavière. Tous deux incarnent donc une résolution esthétique du conflit entre les pôles du pouvoir et de l'esprit. La figure de l'avocat Siegbert Geyer n'est pas retenue dans ces œuvres, et elle est effacée des mémoires de Klenk. L'homme écartelé entre l'esprit et l'action a échoué à entrer dans l'histoire.

19 Tous les complexes d'intrigues tissant la trame du roman de Feuchtwanger sont rapportés à la présence centrale du Land de Bavière. L'enracinement, s'il est synonyme de conservatisme et de réaction, est aussi une forme d'identité, d'humanité, comme en témoignent la figure centrale de Johanna Krain, certains types bavarois positifs, et jusqu'au ministre de la justice Otto Klenk. L'intellectuel apatride Tüverlin conclut avec Johanna Krain une alliance symbolique, sans laquelle son œuvre resterait abstraite, intellectuelle, sans résonance.

20 Rattachée au thème central de l'identité bavaroise, la tragédie de Siegbert Geyer figure celle de l'exclusion du juif. Son impuissance à agir est conditionnée au départ par sa situation d'étranger. L'opposition Bavarois-étranger commande la présentation du juif comme conscience individuelle déchirée, tandis que l'antisémitisme est l'un des thèmes du discours de la haine de tout ce qui n'est pas assimilable à la réalité bavaroise. L'évolution du roman, avec les étapes de la réaction, ne fait que traduire dans les faits l'exclusion dont le principe est formulé au départ.

21 Une des premières scènes $d u$ roman rassemble dans une taverne populaire les personnalités de la scène politique et littéraire bavaroise (60-68). Dans un décor et une atmosphère «bonhomme» (bieder) et «populaire " (volkstümlich), les clivages politiques s'estompent devant la réalité de l'appartenance régionale. L'impression subjective de Geyer, qui se sent étranger à l'atmosphère bavaroise, et isolé même parmi ses amis politiques ("Malgré leurs discours socialistes, ils étaient pareillement englués dans la même idéologie paysanne »), est objectivée par un incident. Lorsque l'avocat critique les élucubrations pseudo-scientifiques d'un vieux professeur munichois sur les «lois biologiques » de Munich, il est immédiatement contredit par le ministre de la culture, qui, négligeant les objections de fond, défend vigoureusement la valeur sentimentale de l'œuvre, avec l'approbation tacite de tous. « Le ministre conclut, sur un ton doux et conciliant, que l'on ne pouvait en vouloir à monsieur le député Geyer de ne pas avoir vraiment le sens de ces choses-là. On n'était accessible à ces sentiments que lorsqu'on était enraciné dans ce sol» (67). C'est ce moment que choisit l'auteur pour évoquer les origines juives de l'avocat, la scène de la taverne agit comme révélateur de son identité juive. 
L'opposition entre l'avocat juif et le ministre bavarois de la justice prend ainsi la forme de l'exclusion de l'étranger. $\mathrm{Si}$, dans les rencontres de personne à personne, les origines juives de l'avocat ne sont pas mentionnées, l'antisémitisme est bien une composante de la mentalité de Otto Klenk. Dans une scène du roman, le ministre traverse sa région en voiture :

Un car d'excursion confortable le dépasse... Il y a beaucoup d'étrangers qui passent maintenant par le pays... Il n'a rien, en soi, contre les étrangers. Possible que les Chinois soient les représentants d'une culture beaucoup plus ancienne et plus raffinée que les Bavarois. Mais lui, il préfère les Knödel et les saucisses aux ailerons de requin... Il n'a pas l'intention de se laisser bouffer par une culture étrangère...

Il pensa soudain à l'avocat Geyer... Il mordit le tuyau de sa pipe. Celui-là, s'il l'a un jour au bout de son fusil, il en fera de la chair à pâté. Logique, droits de l'homme, unité du Reich, démocratie, $\mathrm{XX}^{\mathrm{e}}$ siècle, points de vue européens : du vent. Il souffle par ses lèvres serrées, gronde, tel un animal excité contre son ennemi. Comment ce Geyer qui fait l'important, qui brasse du vide, comment cet arriviste, ce porc de juif, peut-il comprendre ce qui est bien en Bavière et juste pour la Bavière ? Personne ne l'a appelé. Ici, personne ne veut être amélioré. Et quand un crétin pareil vient fourrer son nez, il réussit tout juste à faire tourner la bière.

Le ministre est un homme intelligent, d'une grande science. Excellent juriste, issu d'une famille aisée, de tradition cultivée, qui depuis des générations fournit le pays en hauts fonctionnaires, connaissant l'homme et le bois dont il est fait, il pourrait naturellement, s'il le voulait, rendre justice à Geyer. Mais il ne songe pas à vouloir. (71-72)

Klenk se déchaîne contre l'avocat au moment où les cars de tourisme lui rappellent l'intrusion de la modernité. Mais les processus économiques, les étrangers, sont des entités abstraites et lointaines. L'avocat juif, lui, est l'adversaire désigné, vulnérable par son isolement. L'antisémitisme de Klenk est la réaction de légitime défense contre l'intrus menaçant l'intégrité de la Bavière. Tous les stéréotypes négatifs de l'idéologie conservatrice, anti-occidentaliste et anti-moderniste sont ici dénoncés comme manifestations de «l'esprit juif », avec une rare violence : la République et sa politique centralisatrice, l'universalité des principes de l'Aufklärung, le modernisme. Au nom de l'appartenance au Volksstamm bavarois, Klenk légitime l'exclusion a priori de celui qui ne peut se réclamer du même enracinement, comme il défend une politique fondée non pas sur des principes universels abstraits, mais sur le particularisme (la justice doit être "de la même étoffe que l'Etat lui-même »). Une phrase de Oswald Spengler illustre cette tonalité particulière de l'antisémitisme: «L'homme qui est lié à sa nation par toutes les fibres de son être $a$, en dernière analyse, un rôle positif d'affirmation, même là où il détruit; celui qui, psychiquement, restera toujours un étranger détruit, même lorsqu'il voudrait faire œuvre constructive... L'esprit juif est porteur d'anéantissement, où qu'il intervienne ${ }^{15} »$.

La tragédie familiale reprend au niveau individuel le motif de l'exclusion du juif dont il est victime au plan politique. Son fils, né d'une femme qui a refusé de l'épouser, élevé dans la haine de son père, s'engage aux côtés de Kutzner, et réussit ainsi à paralyser l'action politique de Geyer en le compromettant dans les crimes de l'extrême-droite. Il lui écrit ensuite: «Lui, E., ne pouvait pas s'imaginer que des gens aux idées si différentes puissent être du même sang. Malheureusement, il n'y avait fichtre pas beaucoup de moyens pour éclaircir définitivement cette question. Si, tout de même, il y en avait un... Et il exposait à Geyer le procédé du professeur Sangemeister de Königsberg, en l'exhortant à se soumettre à ce test sanguin » (631-632) ${ }^{16}$. Le reniement de son fils, puis sa mort dans le putsch manqué entraînent l'effondrement définitif de 
Siegbert Geyer, et son exil. La loque humaine que croise Otto Klenk à la fin n'est plus le brillant avocat, mais le juif traqué.

Dans le rôle positif du défenseur des valeurs libérales, Geyer n'est pas différent du type de l'avocat juif des Justizromane de Weimar. Mais il n'est pas réductible à cette seule dimension. Feuchtwanger, lui-même juif assimilé, fort attaché au milieu bavarois, a figuré ici avec intensité la tragédie de l'assimilation, surdéterminée par une triple exclusion : Geyer est rejeté par le Volk bavarois, écarté du pouvoir, renié par son fils. Détaché du judaïsme, qui ne joue plus aucun rôle dans sa conscience individuelle, aspirant à l'enracinement en milieu allemand, il ne peut plus revendiquer sa différence comme identité positive, il la subit comme un stigmate qui le voue à l'exclusion. À quelques années de distance, la retraite de Geyer dans le sud de la France préfigure l'exil des juifs déchus de la citoyenneté allemande.

\section{Schaffer, le juif nationaliste}

Le roman de Ernst von Salomon tire son argument de deux expériences autobiographiques: l'activité militante au service de la cause paysanne ${ }^{17}$, et la fréquentation de milieux intellectuels berlinois, transposées dans l'itinéraire du journaliste Ive. Ancien combattant de la Première Guerre mondiale («Sa patrie était le front ; sa famille, la compagnie ») (25), nostalgique de la camaraderie du front, il végète avant de devenir le journaliste de la cause paysanne au moment du procès de Neumünster, et part ensuite à Berlin pour y chercher des appuis politiques. Succédant au monde de la Ferme, incarnation de la vie organique et de l'enracinement, la Ville s'impose comme l'univers des idées, abâtardi et corrompu. Ive est emporté dans une dérive intellectualiste, sans ligne d'action concrète. La part proprement narrative se trouve réduite dans le roman au profit de la réflexion, ou de longs débats avec des interlocuteurs symboliques : l'activiste, l'intellectuel décadent, l'artiste, le représentant du pouvoir. En ce sens, Salomon, qui cherche à mettre idéalement en place le réseau des forces qui travaillent l'époque, ne fait pas œuvre réaliste ${ }^{18}$.

L'itinéraire de Ive, représentant la révolution conservatrice, figure une quête «romantique » de l'identité allemande, caractérisée par la démesure des aspirations spirituelles, et la violence saluée comme révolutionnaire. Déchiré entre la revendication de la liberté individuelle et le désir d'un ordre supérieur dans lequel il se fondrait entièrement, Ive figure la "conscience faustienne », qui ne trouve pas encore la possibilité historique de s'incarner dans la réalité ${ }^{19}$. La revendication d'un destin allemand se construit sur un double postulat: l'affirmation de la nation allemande comme « élément agissant originel » donne priorité à la vie sur la raison; la notion de " peuple ", conçue comme essentielle différence, s'oppose à l'homme en soi, et donc à la conception libérale dont les institutions républicaines ne sont que la caricature. Dans un contexte général de décadence, le mouvement paysan, spontané, est l'image de la mobilisation pour «défendre la vie elle-même au milieu des puanteurs de la décomposition " (61). Il représente un potentiel révolutionnaire qui peut être mis au service de l'idée nationale allemande, et doit être préservé contre les tentatives de récupération tant des communistes que des nazis.

La revendication d'une spécificité de la vocation allemande ne peut s'affirmer positivement que si elle se traduit concrètement par l'exclusion. Le discours sur la nation est donc, en même temps que l'invocation d'une essentielle et irréductible 
singularité, la légitimation d'un rejet. Dans ce système, le juif a pour fonction d'incarner le corps étranger, non-allemand, contre lequel se mobilise le défenseur de l'idée de nation.

Dans la première partie, sous le signe de la Ferme, le juif n'apparaît pas comme figure individuelle, mais l'antisémitisme est présent. Le cas de Grafenstolz, caricature du petit bourgeois paupérisé ( Malgré les efforts de Grafenstolz, tout allait de travers, et bientôt, il fut tout à fait convaincu que sa malchance était due aux machinations secrètes des juifs, des Jésuites et des Francs-Maçons») (31), trouvant dans l'antisémitisme militant un exutoire à son ressentiment, permet essentiellement de démarquer l'attitude de Ive de « la bavarde activité des bouffons antisémites » (59). La scène où Ive, parti avec un groupe de SA dans une tournée de propagande chez les paysans, lance à l'étudiant chargé d'exposer les buts du mouvement : "Mot d'ordre contre les intermédiaires »:

L'étudiant rit, et déjà il attaquait le sujet. « Les intermédiaires juifs... » Ive l'entendit et il sourit. Lui aussi, dans le Peuple des Campagnes, avait écrit sur les intermédiaires juifs, il avait parlé comme les paysans, et les paysans avaient fait la découverte que dans les provinces du nord le commerce juif n'existait pas. (132 sq., c'est moi qui souligne)

montre que Ive sourit de la bêtise avec laquelle le jeune SA récite sa leçon antisémite, mais sans intervenir, dans le cadre d'une stratégie d'agitation.

L'antisémitisme de Ive est à caractère intellectuel, il est solidaire de ses positions théoriques sur la révolution conservatrice :

Ive était antisémite naturellement, il l'était parce que c'était trop compliqué de ne pas l'être. Partout sur son chemin, il n'avait trouvé les juifs que comme ennemis. C'était une chose curieuse, mais qui ne l'inquiétait pas. Pour lui, leur infériorité était un fait. Leur attitude était inactuelle... Dans beaucoup de conversations, Ive avait pu constater... qu'ils ne pouvaient saisir les choses claires et naturelles; par exemple, il n'avait jamais réussi à leur faire accepter la simple notion de la Ferme... ! Leur primauté indiscutable dans beaucoup de domaines, en particulier dans ceux du commerce, des arts, et de la presse, ... lui semblait seulement conditionnée par le régime; il fallait donc remplacer celui-ci par un nouveau régime, qui par le seul fait d'introduire une échelle de valeurs étrangères à la nature juive - cette échelle de valeurs s'annonçait partout et particulièrement dans la lutte des paysans - devait nécessairement amener la fin de cette primauté désagréable (59).

33 À la différence des réactionnaires, pour qui les juifs incarnent la menace de la modernité, Ive, militant de la révolution conservatrice, les voit comme représentants de l'inactualité du libéralisme. Dans l'Allemagne future, rendue à ses vraies valeurs par la révolution nationale, le libéralisme, corps étranger à sa nature, et avec lui, les juifs, sont voués à disparaître. La résolution du problème politique aura pour conséquence naturelle l'élimination de la présence juive. Le ton dépassionné de Ive, bien différent de l'invective réactionnaire, reflète son assurance, fondée sur une analyse de l'évolution historique en terme de nécessité objective.

Les données observables de la réalité sociologique (la faible présence des juifs en milieu rural, et leur établissement dans les grandes villes sous la République de Weimar) sont ici traduites dans un système de normes, qui valorise la Ferme (incarnation des valeurs de la révolution conservatrice, dont l'évidence relève de l'instinct) contre la Ville, domaine de l'activité juive (le commerce, les arts, la presse), intellectuelle et vénale. Deux des trois types juifs figurés dans la seconde partie du roman sont entièrement 
réductibles à ces stéréotypes négatifs: Salamander, au lendemain des élections triomphales pour le parti nazi, tremble pour son argent et sa personne, et le marchand d'art Jacobsohn est un personnage ignoble.

Seul le troisième, Schaffer, est un véritable interlocuteur de Ive, et surtout, bien différent du stéréotype du juif libéral, il est attaché au principe même d'une révolution conservatrice. Il joue un rôle-clé dans l'initiation intellectuelle de Ive, en l'introduisant dans son cercle hebdomadaire où se retrouvent des hommes de toutes tendances politiques pour débattre des problèmes du jour.

La discussion centrale entre les deux personnages, à l'occasion du succès électoral des nazis en septembre 1930 (140-159), montre les enjeux de l'affrontement. Schaffer tente de convaincre Ive de la sincérité de sa démarche et de proposer à son interlocuteur un credo minimum nationaliste qui formerait une base d'entente : négation du judaïsme comme forme dépassée du nationalisme, rejet de l'idéologie libérale, dénonciation de la pensée réductrice du communisme, affirmation du spirituel contre la pensée technique et le capitalisme, prophétie de la mission civilisatrice de l'Allemagne.

Or pour Ive, l'enjeu du débat n'est pas de dégager un consensus, mais de défendre une position usurpée par un intrus. Il commence par confiner Schaffer dans le domaine des idées, stérile, pour se réserver le sentiment, authentique, «l'appel absolu du sang » et la "conscience de race", propres à légitimer la violence terroriste. Devant les réticences de Schaffer, Ive le taxe de tiédeur et d'esthétisme, pour le renvoyer à la politique parlementaire "Pourquoi n'allez-vous pas au Reichstag?». Puis il procède par retranchements successifs :

38 - Lorsque Schaffer condamne le nazisme comme falsification de la révolution nationale: "On devrait arracher ce mot de nation des bouches insolentes de ces imbéciles !», Ive, par ailleurs porté à mépriser le nazisme, lui dénie le droit de légiférer sur les affaires allemandes : «On devrait... mais qui vous donne le droit d'employer cette formule?»

39 - Lorsque Schaffer analyse l'idée éthique du " socialisme » prussien, don de l'Allemagne au monde, Ive, invoquant la contradiction entre le socialisme et l'âme allemande, développe le thème de «l'attachement naturel..., essentiellement non-spirituel » à la nation, en particulier chez les paysans. La vocation allemande concrète est inséparable d'une terre, et donc inaccessible aux juifs qui, par leur histoire (« Le peuple juif n’a pas de terre »), se trouvent réduits à poser les principes du libéralisme (Schaffer a refusé l'épithète de libéral). Or, ceux-ci sont dangereux pour le peuple allemand "quand on lui a enlevé des terres, quand son existence même est en péril, quand il est vaincu partout excepté dans son noyau intime ».

40 - Il accuse donc Schaffer de se livrer à la "prostitution transcendantale » en proclamant une responsabilité universelle de l'Allemagne tant que celle-ci n'a pas retrouvé son intégrité terrtoriale et tant qu'elle vit dans l'aliénation des villes. La vocation de l'Allemagne doit être en conformité avec l'essence allemande, donc impérialiste, elle doit être définie par les Allemands eux-mêmes, et non par une " exigence juive ", qui « ne peut jamais être valable pour nous (les Allemands) comme commandement primitif ». Le juif converti national se voit refuser le droit de parler de la vocation allemande, converti, il reste juif, et donc libéral à son corps défendant, il ne saurait être Allemand. 
41 Le marquage de l'adversaire comme juif, le thème de l'antisémitisme interviennent aux moments cruciaux où Ive, cessant de respecter les lois du débat intellectuel, se comporte en militant agressif de sa cause. À la première attaque directe "Pourquoi n'allez-vous pas au Reichstag? ", Schaffer accuse une certaine lassitude et ferme les yeux, s'offrant sans défense à Ive, qui le regarde alors avec malaise et pitié (« Mais il a l'air très juif » : c'est la première mention explicite des origines juives de Schaffer). A l'attaque suivante, apparaît le terme d'antisémitisme, suggéré par Schaffer pour expliquer l'hostilité de Ive, mais récusé par celui-ci, qui prétend combattre le juif en tant que défenseur du bastion libéral. Lorsque Schaffer se disculpe de l'accusation de libéralisme et proclame son nationalisme, Ive, troublé malgré lui par sa sincérité, la dévalue parce « qu'elle n'avait pas de centre de gravité proprement dit ». C'est la base de sa grande tirade sur la différence allemande, qui lui permet d'invalider les prétentions du juif sans terre, reléguant l'antisémitisme au rang des problèmes politiques secondaires.

42 L'importance accordée à la figure de Schaffer permet de déceler une ambiguïté de la position de Ernst von Salomon. L'attitude de Ive à l'égard de Schaffer, faite de circonspection ("une amitié prudente»: notons que Schaffer, seul de tous les personnages qui entourent Ive, est exclu de la "camaraderie du front", seul lien authentique), d'estime réticente et d'hostilité fondamentale, est significative par son ambivalence même. Ive, visiblement préoccupé de rendre justice à Schaffer, lui accorde une nette supériorité intellectuelle (un «incomparable art de la discussion", nous sommes à la limite du stéréotype ; la description de la carrière de Schaffer révèle le même caractère tendancieux : c'est l'homme qui a bâti sa situation financière sur une idée publicitaire, niaise de surcroît); mais au nom de «la contradiction de leurs natures ", il va jusqu'à soutenir des positions contraires à ses idées pour ne pas avoir à être d'accord avec Schaffer. Face à l'homme, Ive est visiblement mis en difficulté, et la reconnaissance forcée de la sincérité de son adversaire, voire peut-être la tentation de s'identifier à lui, le dispute un moment à l'hostilité de principe. Salomon ne se contente pas ici de l'expédient de la caricature antisémite (comme il est capable de le faire pour d'autres personnages dans le même roman), l'entretien essentiel entre Ive et Schaffer permet de fixer un point de vue sur le juif, ce qui pose immédiatement le problème de la figuration: nul doute que la tentation existe de faire de Schaffer un véritable personnage. Mais la logique de la révolution conservatrice (les juifs, inactuels, doivent disparaître) est menacée si le juif cesse de figurer l'entité abstraite, l'adversaire par nécessité historique, si une relation personnelle s'instaure. Ive est contraint de mobiliser toutes ses réserves idéologiques pour refouler ses réactions de sympathie ou de gêne ou leur donner un alibi théorique, et c'est cela qui empêche l'émergence d'un personnage ${ }^{20}$. La nécessaire construction du juif comme ennemi s'effectue au prix d'une lourde théorisation, et d'une réduction de l'adversaire à un portrait stéréotypé : Schaffer, subtil adversaire, qui se dit Allemand, nationaliste, prussien, antilibéral, est arbitrairement déclaré juif, non-allemand, libéral. Après cette scène, la figure de Schaffer, un moment proche, s'éloigne, et à la fin, il n'est plus différent des caricatures antisémites de Jacobsohn et de Salamander.

43 Ive se démarque de l'idéologie völkisch et nazie par le désaveu de l'antisémitisme comme programme, sa subordination à la perspective d'ensemble de la révolution conservatrice. On pourrait dire qu'il est anti-juif par système ${ }^{21}$, et non antisémite. Mais cette justification intellectuelle de l'attitude antijuive, corrélative de la banalisation de 
l'antisémitisme réel comme problème secondaire, à résoudre par les gouvernements futurs, occulte la reconnaissance d'une possible interaction entre les deux: l'intellectuel cautionne la démagogie antisémite, et son attitude pourrait bien emprunter un certain nombre de ses caractéristiques à l'antisémitisme le plus grossier. Salomon évacue le problème du juif et de l'antisémitisme, il ne le réfléchit pas. Il nie par système l'actualité d'une problématique dont il refuse de faire un thème de la conscience d'époque.

Ces trois portraits montrent qu'une synthèse de l'image du juif et de l'antisémitisme dans le Zeitroman de la République de Weimar pourrait se construire, non en déployant l'éventail des variantes ou des constantes du portrait « du » juif et de l'antisémitisme, mais en concentrant l'analyse sur quelques complexes représentatifs, où entrent en relation un type de juif correspondant à un type d'antisémitisme qui sont conditionnés l'un par l'autre dans la mesure où ils définissent une problématique homogène. Nous voudrions conclure en reprenant de manière synthétique trois complexes essentiels :

- les facteurs socio-économiques : une domination de l'Allemagne par les juifs?

- les facteurs politiques et idéologiques : un pouvoir juif?

- le problème de l'assimilation : juif et Allemand?

\section{Les facteurs socio-économiques : une domination de l'Allemagne par les juifs?}

Le Zeitroman reflète une réalité socio-économique dont les traits saillants sont: l'absence des juifs en milieu agricole et leur implantation dans les grandes villes; la proportion significative de juifs dans le secteur commercial, ainsi que dans les petites et moyennes entreprises; enfin leur afflux vers les professions libérales. Mais du même coup, il prend acte du divorce entre la situation réelle des juifs, leur importance véritable dans la vie économique, et l'image mythique qu'en donne une propagande agressive.

L'antisémitisme économique est alimenté par quelques grands thèmes mobilisateurs (l'assimilation du juif au grand capital, le mythe du complot des juifs, des jésuites et des Francs-Maçons) qui fonctionnent comme slogans ou fantasmes individuels. Ce sont souvent les figures épisodiques de commerçants juifs qui cristallisent la haine populaire. Un nom à consonnance juive marquée peut servir de signal, comme celui du chapelier munichois Rothschild chez Feuchtwanger. L'antisémitisme peut même fonctionner en l'absence du juif réel (les « intermédiaires juifs » sont inexistants dans certains milieux paysans d'Allemagne du Nord). La figuration de cette variante de l'antisémitisme ne se prête pas, en tout cas, à l'émergence de types juifs, et parallèlement, d'antisémites fortement caractérisés : elle se borne à l'évocation d'une situation de déclassé, et à l'énumération de quelques clichés, dans une intention satirique.

Il y a un abîme entre les juifs réels et l'image mythique du juif omniprésent, incarnation démoniaque du pouvoir de l'argent, travaillant à ruiner l'Allemagne. Le déclin économique relatif des juifs durant cette période s'accorde mal avec les mythes de la propagande antisémite et infirme donc le stéréotype de la «domination " juive, même si l'antisémitisme, qui trouve un large écho dans des couches sociales prolétarisées, chez des individus marginalisés et déséquilibrés, contribue au maintien d'une réelle discrimination. 
Mais du même coup, il semble que le thème juif, vu sous cet angle, s'épuise très vite dans le Zeitroman weimarien. La différence est très nette avec les romans de la période précédente, si l'on songe par exemple que le Soll und Haben de Gustav Freytag repose tout entier sur la peinture d'une évolution économique dans laquelle le juif joue un rôle négatif: Ehrenthal dépouille de ses biens le baron Rothsattel, symbole de la noblesse ancienne ruinée par le capitalisme naissant. La carrière exemplaire du héros bourgeois Wohlfahrt contraste avec l'enrichissement frauduleux du juif Itzig. Dans le Zeitroman de la République de Weimar, le juif n'incarne plus le péril économique qui menace la survie de couches sociales entières, mais le thème de l'antisémitisme économique, du même coup, ne se prête plus guère à la figuration, et devient marginal.

\section{Les facteurs politiques et idéologiques : un pouvoir juif ?}

En revanche, les bouleversements politiques qui accompagnent l'écroulement de l'empire et l'établissement de la République, l'instabilité du régime, favorisent l'émergence d'un thème nouveau. Le juif se profile comme l'adversaire idéologique et politique, le fossoyeur de l'ordre ancien. Le couple «allemand-juif » exemplaire n'est plus figuré, comme dans Soll und Haben, par l'opposition entre les valeurs de la naissance et celles de l'argent (Rothsattel-Ehrenthal), le travail honnête et l'usure (WohlfahrtItzig), mais par des antagonismes idéologiques et politiques: le juge réactionnaire et la communiste, le Bavarois particulariste et l'avocat social-démocrate, le militant de la révolution conservatrice et le juif « inactuel », agent de la décadence de l'Allemagne.

Le Zeitroman weimarien, qui enregistre l'insignifiance relative des juifs dans la vie économique, reflète, à des degrés divers, leur importance accrue dans la vie publique. Dans le domaine culturel et artistique, des figures épisodiques sont associées à la peinture des milieux décadents (pensons au marchand d'art Jacobsohn dans la Ville, symbole de la lubricité, du pouvoir de l'argent, de la corruption des valeurs créatrices). Mais là aussi, la prégnance des stéréotypes est trop forte pour favoriser la création de personnages.

51 C'est dans le domaine politique que l'on trouve les possibilités les plus grandes de figuration de ce thème, ce qui ne nous étonnera pas, vu l'importance de l'actualité politique dans le Zeitroman weimarien. Il apparait que le stéréotype du " pouvoir juif ", à l'égal du stéréotype de la "domination économique » des juifs sur l'Allemagne, est fort éloigné de la réalité observable. La plupart des auteurs, en effet, thématisent le triomphe de la réaction ou la montée d'un nouvel extrémisme, et la faible résonance tant des forces libérales que de la gauche radicale, où se retrouvent majoritairement les juifs (pensons à Genia Lazar dans la gauche militante et révolutionnaire, à Siegbert Geyer député social-démocrate); notons pourtant que le cas de Schaffer, nationaliste, illustre une variété qui est l'indice du degré d'assimilation des juifs allemands. Les personnages juifs incarnent de manière privilégiée la légalité républicaine, l'idéal de justice sociale, les valeurs libérales (même le juif nationaliste est combattu comme libéral !), le changement radical. Ce type de personnage juif est le plus souvent l'homme du discours, du plaidoyer, du débat intellectuel, jamais celui de la Realpolitik. Il lui manque la dimension d'un homme d'État, comme en témoigne son absence de prestance physique, opposée à la carrure des hommes qui détiennent la réalité du pouvoir (le ministre bavarois Klenk). 
52 Le Zeitroman reflète l'explosion formidable de l'antisémitisme politique dont les thèmes majeurs se définissent au cours de la Première Guerre mondiale, relayant les motifs économiques et racistes, dominants à la fin du siècle dernier. Ce nouvel antisémitisme, fondé sur l'assimilation du juif à la nouvelle République et au bolchevisme, utilisé tant par les anciennes classes dirigeantes réactionnaires que par les courants nouveaux de l'extrême-droite, devient un moyen privilégié de manifester son hostilité au régime démocratique. Notons ici que les problèmes de figuration sont les mêmes que dans le cas de l'antisémitisme à dominante économique, et que l'importance de la satire (correspondant au point de vue de l'intelligence libérale discréditant la bêtise et la vulgarité de son adversaire) est peu propice à la compréhension de la dimension menaçante du phénomène.

\section{Le problème de l'assimilation : juif et Allemand?}

À des degrés divers, le sort des personnages juifs qui figurent dans le Zeitroman weimarien est marqué par l'exclusion. Toutefois, la comparaison avec d'autres types sociaux rejetés ou marginalisés ferait apparaitre la spécificité du fait juif. Qu'il s'agisse de chômeurs, d'intellectuels prolétarisés, de femmes déçues par l'émancipation, tous peuvent formuler, pour eux ou pour d'autres, la conscience d'un destin d'époque, et s'identifier ainsi comme type social. Le personnage juif est le seul qui ne puisse pas parler avec d'autres de sa condition d'exclu, et ne trouve dans aucun personnage le reflet d'une expérience semblable de discrimination. Il en résulte une difficulté essentielle à se définir lui-même et à se percevoir comme juif.

La solitude du personnage juif dans le Zeitroman est conditionnée par l'absence d'un environnement traduisant la permanence de références juives. À la différence des romans de la période précédente, qui montrent le juif dans son milieu familial (la famille Ehrenthal dans Soll und Haben), le personnage du Zeitroman weimarien est le type du juif assimilé, qui se caractérise par la rupture avec le judaïsme comme insertion dans une communauté familiale et religieuse. On comparera sur ce point précis l'itinéraire du juif Süß avec celui de types sociaux tels que Siebgert Geyer. La disparition de l'environnement juif n'a pas pour contrepartie la réalité d'un enracinement allemand. Schaffer, renonçant à l'idée nationale juive pour épouser celle de la mission éthique de l'Allemagne, est rejeté du côté du libéralisme, sous-produit d'un judaïsme privé de «terre». Seules des circonstances tragiques, telles que la proximité de la mort, permettent aux personnages de ressaisir un fragment de leur identité juive, encore est-ce moins la réappropriation positive d'une tradition vivante que le constat d'un échec. La rupture se consomme entre judéité et judaïsme.

Du fait que la République de Weimar crée, du moins en théorie, les conditions de l'assimilation définitive, l'image du juif que réfracte le Zeitroman se normalise. Le juif allemand tend à se définir par son rôle et son engagement, et non plus au premier chef par ses origines juives. Il n'est plus figuré comme un criminel ou un parvenu, il n'est plus le personnage uniment noir des romans du siècle précédent. Il peut être présenté et perçu par le lecteur (à défaut d'être tel aux yeux d'autres personnages dans le roman) comme une figure positive. Un indice sûr de cette évolution dans la figuration du juif est la tendance à la régression des stéréotypes dans la caractérisation, physique en particulier. Mais la faiblesse de la tradition libérale à laquelle il doit son intégration dans la société allemande, la permanence des préjugés antisémites hérités de l'époque 
précédente, la mobilisation de la réaction et de l'extrême-droite, recréent les conditions d'une discrimination réelle, ce qui se traduit par la difficulté de figurer le thème juif. Malgré tout, le Zeitroman weimarien, en restituant une perspective contemporaine sans correction ni ajustement a posteriori, nous invite à ne pas réduire la complexe réalité de Weimar à une dérive vers l'époque nazie.

\section{BIBLIOGRAPHIE}

Nous citons les ouvrages étudiés d'après l'édition suivante :

Ottwalt Ernst : Denn sie wissen, was sie tun. Ein deutscher Justizroman, Berlin, Verlag europäische ideen, 1977 (reprint de la lre édition, Berlin, 1931).

Feuchtwanger Lion : Erfolg, Francfort/M ; Fischer TB, 1983 (lre édition, Berlin, 1930).

Salomon Ernst von : La Ville, traduction française de N. Guterman, Paris, 1933 (lère édition, Die Stadt, Berlin, 1932).

\section{NOTES}

1. - Cf. le livre de Pierre Angel, Le personnage juif dans le roman allemand, 1855-1915. La racine littéraire de l'antisémitisme outre-Rhin, Paris, 1973. Cet ouvrage a été le premier, en France, à montrer l'intérêt d'une étude de l'image littéraire du juif. A partir de romans écrits durant la période critique qui voit la percée de l'antisémitisme et sa constitution en parti politique influent, il met en évidence la distorsion par laquelle le juif imaginaire se substitue au juif réel et l'interprète comme indice et véhicule de la mentalité antisémite que ces œuvres ont contribué à diffuser et à fixer dans ses grands traits.

2. - H.A. Winkler, «Die Deutsche Gesellschaft der Weimarer Republik und der Antisemitismus» in Die Juden als Minderheit in der Geschichte, éd. par B. Martin \& E. Schulin, dtv, 1982.

3. - Sur ce point, on se rapportera par exemple à l'analyse de Donald Niewyk, The Jews in Weimar Germany, Manchester, 1980.

4. - On ne s'étendra pas ici sur la «conscience d'époque " propre à la République de Weimar, suffisamment connue maintenant, notamment par les travaux du Groupe de Recherches sur la Culture de Weimar, dirigé par Gérard Raulet, auxquels nous renvoyons. Il faudrait examiner dans le détail les remaniements du genre du Zeitroman entraînés par l'émergence d'une conscience d'époque essentiellement négative.

5. - Le Zeitroman est bien cependant une «forme de narration historique " (cf. Die Deutsche Literatur in der Weimarer Republik, éd. par Wolfgang Rothe, Stuttgart, Reclam, 1974).

6. - Dans le travail magistral qu'il a consacré à l'étude du roman historique, Georg Lukacs relève la difficulté d'auteurs tels que Heinrich Mann et Feuchtwanger à figurer des personnages concrets et vivants dans le cadre de ce genre, devenu, à l'époque de Weimar, le véhicule de thèses morales et philosophiques.

7. - L'œuvre reproduit les conclusions de l'analyse sociologique du social-démocrate Ernst Fraenkel, Zur Soziologie der Klassenjustiz, Berlin, 1927. 
8. - En ce sens, on pourrait définir l'itinéraire de Dickmann comme anti-Bildungsroman. La comparaison avec Der Untertan nous semble s'imposer.

9. - Le point culminant du roman coïncide avec le «Leipziger Bluturteil», à l'issue du procès de la Tcheka. Sur la dénonciation de l'institution judiciaire dans la littérature de la République de Weimar, on se reportera à l'ouvrage de Klaus Petersen, Literatur und Justiz in der Weimarer Republik, Stuttgart, 1988.

10. - Nous ne développerons pas ici davantage les raisons de ces affinités électives entre les juifs et les forces de gauche. Nous renvoyons sur ce point à l'ouvrage de Michael Löwy, Rédemption et utopie. Le judaïsme libertaire en Europe centrale, Paris, 1988.

11. - Que la misogynie soit bien un constituant de la mentalité réactionnaire, ressort à l'évidence du livre de Klaus Theweleit, Männerphantasien. L'étude de Rita Thalmann, Etre femme sous le Troisième Reich (Paris, 1982) montre qu'à l'époque suivante, l'assujettissement de la femme à la domination du mâle est un des aspects essentiels de la doctrine et de la politique des nazis.

12. - Deux variantes de l'antisémitisme, qui ne sont pas retenues par l'analyse marxiste, sont en revanche fort bien perçues par l'écrivain libéral : celui que l'on trouve en milieu populaire (chez Fallada, par exemple, un sentiment anti-juif diffus) ou celui qui serait rendu possible par le communisme lui-même, qui assimile le juif au grand capital (cf. les scènes de la révolution spartakiste chez Hermann Glaeser). Cela dit, le thème juif est totalement absent des romans prolétariens. La lucidité d'Ottwalt est à mettre en rapport avec son expérience des milieux réactionnaires, thématisée dans le roman Ruhe und Ordnung, Berlin, 1929.

13. - Entre autres, l'existence des Volksgerichte bavarois, qui se signalent dans la répression de la gauche, et le scandale des conditions de détention, d'après les récits autobiographiques de Max Hoelz, Erich Mühsam, Ernst Toller.

14. - Cf. Synnöve Clason, Die Welt erklären. Geschichte und Fiktion in Lion Feuchtwangers Roman « Erfolg», Stockholm, 1975.

15. - Cité par Pierre Vaydat dans «L'antisémitisme de la Révolution conservatrice " (in Annales du C.E.S.E.R.E., 1980, p. 72), auquel nous renvoyons pour l'analyse approfondie des variantes de l'antisémitisme dans les différents courants qui se rattachent à la Révolution conservatrice.

16. - On aura reconnu ici la polémique contre les théoriciens du racisme. La «Königsberger Blutsprobe » fait penser aux expériences du médecin Gsell, par exemple. Cet antisémitisme biologique sous-tend le roman de Arthur Dinter, Die Sünde wider das Blut (Leipzig, 1918).

17. - Le «Landvolkbewegung » s'est développé dans le Schleswig-Holstein à partir de 1929, dans un contexte marqué par les difficultés économiques, en réaction à l'imposition du gouvernement. Les épisodes de ce conflit ont été repris par Fallada dans son roman Bauern, Bonzen und Bomben, à projet nettement réaliste.

18. - Si l'on s'en tient à la définition du Zeitroman comme œuvre dont l'enjeu est la lisibilité de l'époque, l'ensemble des personnages figurant les multiples aspects d'une conscience d'époque vécue au niveau de la confusion, on n'hésitera pas à rattacher La Ville à ce genre, bien que l'on ne puisse plus guère parler d'action romanesque dans la seconde partie.

19. - Le terme est emprunté à Oswald Spengler, dont on peut retrouver les idées directrices (dans Prussianité et Socialisme, de 1919, par exemple) dans le discours de Ive.

20. - On relèvera la similitude du traitement de la figure de Rathenau dans Die Geächteten: contraint de rendre justice à l'homme d'Etat ("ce pathos était authentique »), le narrateur ne peut que le mettre à distance pour éviter le piège de la sympathie, de l'admiration, de l'identification possible à un juif.

21. - La possibilité d'une amitié entre un représentant de la droite nationaliste et un juif est illustrée par la relation entre Léo et Konrad dans L'ami retrouvé de Fred Uhlmann. Au moment du départ de Léo en 1933, Konrad exprime sa tristesse qu'il n'y ait pas, momentanément, de place pour les juifs dans l'Allemagne nouvelle, et son espoir de voir son ami revenir plus tard. 


\section{RÉSUMÉS}

La représentation d'une réalité juive normalisée, diversifiée, dans le Zeitro man de la République de Weimar, contraste fortement avec le portrait caricatural « du » juif démoniaque, marqué par les stéréotypes de l'antisémitisme économique, omniprésent dans les romans de l'époque précédente. On peut s'étonner cependant de la place assez réduite qu'occupe le thème juif dans ces œuvres. En conséquence, nous envisagerons ici l'image du juif et de l'antisémite moins sous l'aspect de la vérité documentaire que sous celui de la figuration littéraire. Nous indiquerons au passage l'importance du genre comme cadre de figuration en démarquant le roman d'époque, qui figure l'actualité dans sa dimension problématique, non encore résolue, du roman historique. L'analyse de trois personnages juifs dans leur contexte romanesque (Ottwalt: Denn sie wissen, was sie tun; Feuchtwanger: Erfolg; Salomon: Die Stadt) permet de voir l'importance croissante des motifs politiques, et nous amène à constater qu'il n'y a pas « le » juif, ou « l' » antisemitisme, mais que la construction d'un "complexe de faits » juif dans le roman s'opère en fonction d'une perspective idéologique donnée, qui conditionne le degré de figuration des motifs juifs particuliers, et leur organisation dans un cadre d'interprétation.

Im Gegensatz zum dämonisierten, von wirtschaftlichen Stereotypen geprägten Zerrbild «des» Juden, charakteristisch für die Romane der vorausgehenden Epoche, dokumentiert der Zeitroman der Weimarer Republik eine weitgehende Normalisierung und Differenzierung des Judenbildes. Erstaunlicherweise nimmt aber das jüdische Thema im Zeitroman der Weimarer Republik einen eher geringen Platz ein. Wir wollen also das Bild des Juden und des Antisemiten hier weniger auf seinen dokumentarischen Wert hin prüfen, als vielmehr im Hinblick auf die Frage seiner literarischen Gestaltung untersuchen. Dabei wird die Bedeutung des Genres als Gestaltungsrahmen angedeutet; in dieser Hinsicht wird der Zeitroman, der sich mit der Aktualität als ungelöster Problematik befaßt, gegen den zeitgenössischen historischen Roman abgegrenzt. Die Analyse von drei jüdischen Figuren in ihrem jeweiligen Romankontext (Ottwalts Denn sie wissen, was sie tun; Feuchtwangers Erfolg; Salomons Roman Die Stadt), die der wachsenden Rolle der politischen Motive Rechnung trägt, soll darauf hinweisen, daß es nicht «den» Juden oder «den» Antisemitismus gibt. Die Gestaltung eines jüdischen «Tatsachenkomplexes» im Roman erfolgt nach einer ideologisch bedingten Perspektive, was die Hervorhebung bzw. Unterschätzung der einzelnen jüdischen Motive und ihre Organisation in einem Deutungsrahmen bestimmt.

\section{AUTEUR}

\section{ANNE LAGNY}

Université Charles de Gaulle - Lille III 